\title{
Pseudospirochaetosis of the urinary bladder
}

\section{S Chitale, N Burgess, T H W Barker, P F Roberts}

J Clin Pathol 2005;58:437-438. doi: 10.1136/icp.2004.020529

This report describes an elderly patient with urinary symptoms who showed surface colonisation of the transitional mucosa of the bladder by an unusual haematoxophilic microorganism superficially resembling the "blue fuzz" seen in colonic biopsies showing intestinal spirochaetosis. Special stains showed that the organisms were Gram and Giemsa positive, weakly argyrophilic, and Ziehl-Nielsen negative. Immunostains were negative for Helicobacter pylori and electron microscopy revealed curious curved bodies, which were difficult to classify. Therefore, this condition was described as pseudospirochaetosis of the urinary bladder. The urinary symptoms regressed on treatment with ciprofloxacin. The clinicopathological relevance of these findings is discussed in the report.

A n 82 year old woman presented with irritative lower urinary tract symptoms. She had bilateral large simple renal cysts managed by ultrasound guided aspiration in the past. A follow up computed tomography scan for the renal cysts revealed an obstructing lesion at the right vesico ureteric junction. Full blood count and renal function were normal. Midstream urine showed sterile pyuria and heavy mixed bacterial growth of contaminants. Cystoscopy revealed generalised cystitis. Retrograde pyelography ruled out vesico ureteric junction obstruction or urothelial neoplasia. Bladder biopsies confirmed follicular cystitis without urothelial atypia or carcinoma in situ.

The adjacent urothelium showed surface colonies of spirochaete-like haematoxophilic organisms in the form of a "blue fuzz" (fig 1), similar to that seen in human intestinal spirochaetosis (HIS). Special stains confirmed these to be Gram positive cocci or coccobacilli. Warthin Faulkner staining for spirochaetes was focally positive and Giemsa staining was also positive. Immunohistochemical staining for Helicobacter pylori was negative. Electron microscopy (EM) revealed short rod shaped bacilli on the urothelial surface with a helical and curved morphology (fig 2). These highly electron dense bodies on the EM pictures had appearances suggestive of the microsporidium, Enterocytozoonbieneusi, but also had features suggestive of a curved or helical morphology (Dr R Owen, Mr J Williams, and Mr D Ellis; personal communication, 2004). The patient showed significant symptomatic improvement with ciprofloxacin. Three months postoperatively she remained symptom free.

\section{DISCUSSION}

HIS as a result of bowel colonisation by Brachyspiraaalborgi and Brachyspira pilosicali ${ }^{1}$ is a histological diagnosis made on rectal or colonic biopsies from patients investigated for lower gastrointestinal tract symptoms. ${ }^{2}$ The presence of spirochaetes on the surface of the colonic mucosa produces a characteristic "blue fuzz" in routine haematoxylin and eosin staining. It is seen in association with ulcerative colitis or adenomas of the colon, and has been associated with

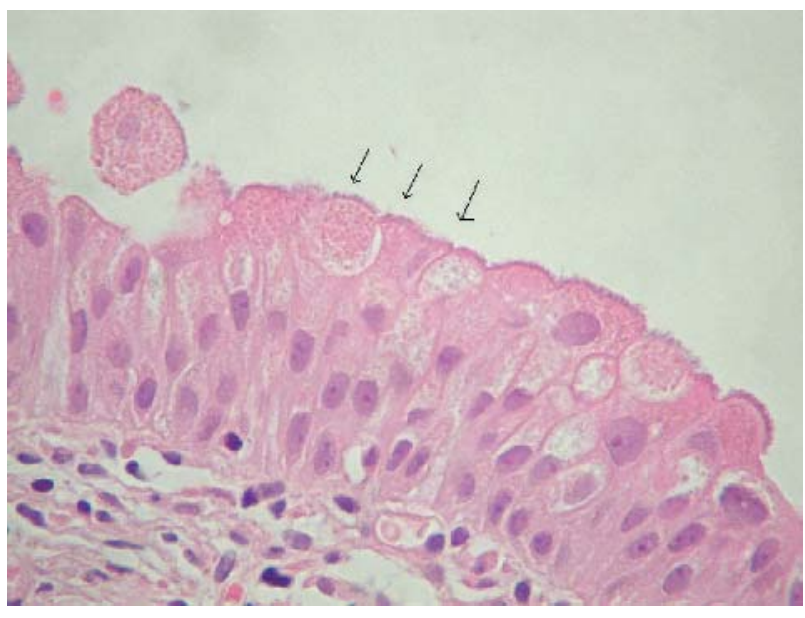

Figure 1 Finely granular haematoxyphilic microorganisms coating the surface of the urothelium (haematoxylin and eosin stain; original magnification, $\times 250$ ).

homosexual activity, the so called "gay bowel syndrome". It has also been seen in asymptomatic patients. It can affect the entire colon but not the terminal ileum. Brachyspira pilosicoli has been found in the faeces of $30 \%$ of people from developing countries including Australian Aborigines, HIV positive patients, and male homosexuals, whereas $B$ aalborgi has been isolated from a patient with HIS in Denmark and is the most common spirochaete species in Western populations. ${ }^{3}$ The pathogenic mechanism for diarrhoea in these cases has been attributed to pathological cell surface changes and the presence of intracellular bacteria in the colonic cells. Symptoms resolve on treatment with metronidazole, ${ }^{4}$ although they can also improve without active treatment. ${ }^{5}$ The prevalence of human intestinal spirochaetosis in Norway has been reported to be in the range of $2.5-3.0 \%$ in colorectal resections, with a significant male predominance (70-75\%). ${ }^{6}$ Significant regional variation in the diagnosis of HIS within Norway was noted as a result of interobserver variation. HIS is therefore considered in the differential diagnosis of patients with chronic diarrhoea and lower gastrointestinal symptoms, although its putative role in relation to these symptoms is still controversial.

Animal studies have shown that the urinary bladder is a consistent source of Lyme disease spirochaetes Borrelia burgdorferi in both experimentally infected and naturally exposed rodents. ${ }^{78}$ Culture of the bladder and urine has been effective in isolating the organisms and confirming the diagnosis. There have been isolated reports of $B$ burgdorferi being found in the human bladder and urine from patients with Lyme disease.

Abbreviations: EM, electron microscopy; HIS, human intestinal spirochaetosis 


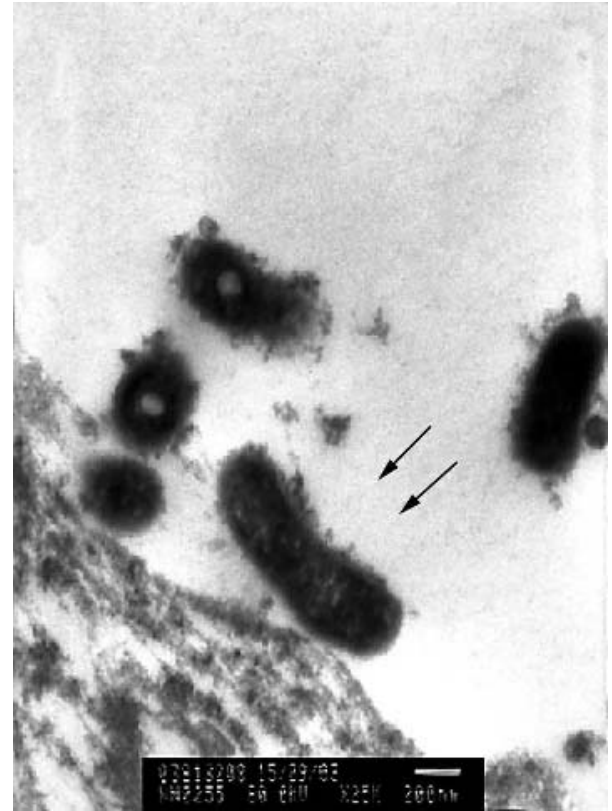

Figure 2 Postfixed electron microscopy preparation (original magnification, $\times 25000$ ) showing cocci and coccobacilli on the surface of the urothelium.

"The mechanism of involvement of the urinary bladder by spirochaetes at present remains unclear, its prevalence unknown, and its importance as yet not fully understood"

In our case, the presenting symptoms were suggestive of urinary tract infection, and the endoscopic appearances agreed with a diagnosis of chronic cystitis, as did the histological picture, which showed "blue fuzz" on the surface of the urothelium, very strongly suggestive of vesical colonisation by spirochaetes. These organisms were unlikely to have been spread by contaminated endoscopes, because the patient had not undergone previous invasive investigations. The organisms could of course have travelled up the urethra from faecal contamination. The special stains showed Gram positive bacilli, which stained focally for spirochaetes ( silver stain), but negatively for helicobacter specific immunostaining. The EM appearances proved difficult to interpret. It was not clear whether they represented a form of spirochaetes or a form of microsporidia. In these circumstances, we have decided to call the condition pseudospirochaetosis.

In patients with recurrent urinary tract infections, chronic cystitis, or sterile pyuria, it may be prudent to look for unusual causes of infections, such as these, and consider them in the differential diagnosis on the same grounds as "HIS" in patients with persistent lower gastrointestinal symptoms and unexplained diarrhoea. The mechanism of involvement of the urinary bladder by spirochaetes at present
Take home messages

- We describe a patient with urinary symptoms who was infected by organisms that resembled spirochaetes but were difficult to classify

- The electron microscopy appearances were difficult to interpret and it was unclear whether they represented a form of spirochaetes or microsporidia, so we decided to call the condition pseudospirochaetosis

- Such unusual causes of infection should be sought and considered in the differential diagnosis in patients with recurrent urinary tract infections, chronic cystitis, or sterile pyuria

remains unclear, its prevalence unknown, and its importance not fully understood.

\section{ACKNOWLEDGEMENTS}

Thanks to Dr R Owen, head of the helicobacter and campylobacter reference unit, SRMD-LEP, Health Protection Agency, London, UK and Dr J Williams and Dr D Ellis, Department of Infectious and Tropical Diseases, London School of Hygiene and Tropical Medicine, London, UK.

\section{Authors' affiliations}

S Chitale, N Burgess, Department of Urology, Norfolk and Norwich University Hospital NHS Trust, Colney Lane, Norwich NR4 7UY, UK T H W Barker, P F Roberts, Department of Histopathology, Norfolk and Norwich University Hospital NHS Trust

Correspondence to: Mr S V Chitale, Department of Urology, Norfolk and Norwich University Hospital, Colney Lane, Norwich NR4 7UY, UK; chitalenorwich@aol.com

Accepted for publication 24 August 2004

\section{REFERENCES}

1 Korner M, Gebbers J. Clinical significance of human intestinal spirochaetosis-a morphologic approach. Infection 2003;31:341-9.

2 Willen R, Carlen B, Cronstedt J, et al. Intestinal spirochaetosis of the colon diagnosed with colono-ileoscopy and multiple biopsies. Endoscopy $1985 ; 17: 86-8$

3 Mikosza AS, Hampson DJ. Human intestinal spirochaetosis: Brachyspira aalborgi and/or Brachyspira pilosicoli? Anim Health Res Rev $2001 ; 2: 101-10$.

4 Rodgers FG, Rodgers C, Shelton AP, et al. Proposed pathogenic mechanism for the diarrhoea associated with human intestinal spirochaetes. Am J Clin Pathol 1986;86:679-82.

5 Lo TC, Heading RC, Gilmour HM. Intestinal spirochaetosis. Postgrad Med J 1994;70:134-7.

6 Lindboe CF. The prevalence of human intestinal spirochaetosis in Norway. Anim Health Res Rev 2001;2:117-19.

7 Czub S, Duray PH, Thomas RE, et al. Cystitis induced by infection with Lyme disease spirochaete, Borrelia burgdorferi, in mice. Am J Pathol 1992;141:1173-9.

8 Schwan TG, Burgdorfer W, Schrumpf ME, et al. The urinary bladder, a consistent source of Borrelia burgdorferi experimentally infected white-footed mice (Peromyscus leucopus). J Clin Microbiol 1988;26:893-5. 